\title{
THE DIFFICULTIES AND DANGERS OF THE MULTIPARA
}

\author{
By \\ A. LEYLAND ROBINSON, M.D.(Lond.), F.R.C.S.(Eng.), F.R.C.O.G. \\ (Professor Obst. and Gyn. University of Liverpool, etc.)
}

It cannot be denied that a woman must have borne a child before her capacity for childbearing can be assessed, and that in respect of prognosis the multipara starts with an immense advantage over the primigravida. Moreover, multiparae represent the survival of the fittest primigravidae, for some women do not survive their first labour, and others are damaged and rendered less fit for further childbearing, or actually incapable of it. The fate of the multipara is therefore intimately wrapped up in the welfare of the primigravida, and the antenatal treatment of the multipara begins or should begin during her first pregnancy.

This paper is not concerned with the general methods of diagnosis and treatment, for these are essentially the same for all cases, irrespective of parity, but rather with the particular influences that are brought into play by the parity and age of the patient. Before discussing these influences in some detail it is important to note that certain general distinctions must be drawn between the first reproductive cycle and all subsequent ones, and that excessive parity sets up changes in parous women which clearly differentiate them into two groups-the young and the old multipara.

The first main difference between the primigravida and the multipara concerns prognosis, for which purpose all pregnant women may be divided into.three groups-(I) the apparently normal-much the largest group; (2) the grossly abnormal-a small, indeed the smallest, group; and (3) the indeterminate or doubtful type who for various reasons cannot be placed with certainty in either of the other groups.

Firstly, the patient whose capacity for reproduction is known from her past record may be classified as normal or abnormal with fair ease, and the doubtful group of multiparae is a very small one. The primigravida, on the other hand, cannot be classified in this simple.way, for one cannot exclude the possibility of the onset of toxaemia from any first pregnancy (however healthy the patient may be), and the behaviour of the uterine muscle during labour cannot be foreseen with certainty: hence there is always an element of doubt (small, perhaps, but inescapable) in regard to the fate of the first reproductive cycle, and therefore all primigravidae should logically be placed in the doubtful category. Secondly, it is beyond dispute that the mortality and morbidity of childbearing is highest among primigravidae of all age groups, and lowest among young multiparae. There are several reasons for this important difference. The knowledge gained during the first pregnancy and labour demonstrates what a patient can and cannot do, and thus indicates the need for special care on subsequent occasions. The first cycle normally induces changes which in various ways render future childbearing less hazardous: the multipara is easier to examine, and therefore diagnosis is more accurate, and the mode of treatment more clearly indicated. Labour itself, other factors being constant, is shorter and easier owing to the elimination of the resistance of the soft parts, whilst uterine inertia and incoordination are much less common in young healthy multiparae. Thirdly, excessive parity introduces changes which serve to distinguish not only the primigravida from the multipara, but also young multiparae from old ones, whilst advancing age brings with it bodily changes that enhance the effects of parity and increase the risk of childbearing. The age factor may operate independently of parity, but in general, primigravidae are younger than multiparae, and excessive parity is rarely seen except among the higher age groups, when it produces the clinical type known as the "Old Multipara."

In the light of these general principles one may discern four types of parous women-(I) The normal multipara; (2) the abnormal multipara; (3) the multipara with a bad obstetric history; (4) the old multipara.

\section{GROUP 1.-THE NORMAL MULTIPARA.}

Given a satisfactory past history, good health, and a normal presentation, these women require the minimum of attention-they should attend an antenatal clinic where arrangements should be made for their delivery at home unless the home conditions are unsatisfactory. Should 
such patients develop signs of toxaemia, an abnormal presentation or other evidence of abnormality, they must be transferred into the second group and treated accordingly.

\section{GROUP 2.-THE ABNORMAL MULTIPARA.}

Multiparae who are frankly abnormal-whatever the type of abnormality may be, are unsuitable for ordinary domiciliary supervision: they require the type of care provided at a consultative antenatal clinic, and admission to hospital for special investigation and treatment. The general methods of dealing with obstetric abnormalities are common to multiparae and primigravidae alike, and they require no special comment in a paper dealing with the particular needs of the multipara-it is sufficient to note that a woman is easier to treat as a multipara because her past experiences provide a standard of performance and of response to treatment which was denied to her as a primigravida.

\section{GROUP 3.-THE MULTIPARA WITH A HISTORY OF ABNORMALITY.}

The woman who has had an abnormal pregnancy or a difficult labour as a primigravida does not necessarily get into trouble on a subsequent occasion, but she may do so, and her treatment and disposal require special consideration.

\section{History of Dystocia.}

The practitioner called upon to deal with a multipara giving a history of dystocia should first ascertain the cause and degree of the previous difficulty from the obstetrician who was in charge of the case and obtain from him (or her) an opinion as to the functional capacity of the birth canal and the treatment appropriate to the condition. If these details are available it should not be difficult to determine the correct line of treatment on the present occasion, and where the disproportion was previously slight the case may be regarded as suitable for domiciliary treatment so long as the practitioner has the necessary skill and experience, and the surroundings are suitable to the simple manœuvres that may be necessary. A woman, for example, giving a history of a difficult forceps comes up for antenatal examination, and states she wishes to be delivered at home. Enquiry elicits the facts that the first baby weighed $8 \mathrm{lbs}$., the maternal soft parts were rigid, but the disproportion was slight and due to non-recurrent factors. Under these circumstances, so long as the presentation is a normal vertex and the child not unduly large, no difficulty need be anticipated, and the patient may be reasonably dealt with at home. Alternatively, the induction of premature labour might be recommended as a preventive measure-and there can be no reason against carrying out this simple procedure at home. When, however, the degree of dystocia at the first labour is reported as having been severe, it is unwise to treat the patient at home, even if the mechanical relationships between mother and foetus appear to be more favourable on the present occasion. It is true that many cases which give a history of severe difficulty in the past may have an easy labour and indeed may not require instrumental help, but there is always a doubt about such cases, and it is much wiser to admit them to hospital, where a "trial labour" can be carried out amid surroundings which enable instrumental or operative methods to be employed if they are called for. This rule applies with especial force to women upon whom Caesarean section has been practised, but it is not always recognised, and the writer has known of many patients who have been allowed to remain at home for labour after'a Caesarean section has been performed on a previous occasion. This procedure is quite indefensible, for it is not fair to the operator, much less to the patient, to allow such a case to go into labour under conditions in which rupture of the scar might occur before help could be obtained. The doctrine "once a Caesar always a Caesar" is not acceptable to all clinicians, but powerful arguments may be advanced in its favour when the operation has been performed upon the patient as a primigravida. The primigravida delivered by this route escapes laceration of the cervix and injury or over-stretching of the pelvic and perineal supports, and is unlikely to develop prolapse, pelvic cellulitis, and probably carcinoma too, unless delivery per vias naturales is permitted at a subsequent labour, when the immunity conferred by the operation is lost. Moreover, a woman delivered by Caesarean section as a primigravida is still a primigravida from the point of view of the stress of natural delivery on the second occasion, so that the strain on the scar is considerable and rupture is always a possibility. When the Caesarean operation is performed upon a woman whose tissues have already sustained the brunt of natural delivery, these arguments in favour of repeating the operation on a subse- 
quent occasion lose their cogency, and similarly there is much less risk of rupture of the scar if delivery takes place per vias naturales. It may therefore be permissible to allow such patients to deliver themselves under special controlled conditions in hospital, but in no circumstances should they be allowed to deliver themselves elsewhere.

\section{History of Malpresentation.}

When dealing with malpresentations two factors have to be taken into account.

(a) The mechanical difficulties involved in the malpresentation itself, e.g. R.O.P., which means a deflexed head and mechanical disadvantage owing to the larger diameter presented to the pelvis.

(b) The significance of the underlying cause, which may greatly outweigh the importance of the malpresentation (Contracted pelvis).

In the primigravida, where examination is impeded by the nulliparous state of the pelvic muscles and where the functional capacity of the pelvis is as yet unknown, it is not so easy to confirm the diagnosis, to determine the underlying cause, or to assess the degree of disproportion created by the malpresentation. In the multipara, when the clinician has access to the previous history, it should be comparatively easy to establish these facts and to choose an appropriate method of dealing with the case-(i) allowing the presentation to persist . . . breech in a normal multipara, (ii) conversion to a better presentation . . . breech to vertex, or (iii) removal to hospital . . . transverse lie with contracted pelvis. Special significance attaches to malpresentations which are known to have occurred in a previous pregnancy, and to those which recur in the same pregnancy after they have been corrected. Such recurrence strongly suggests a definite cause, such as contracted or deformed pelvis, and although the availability of the past history facilitates the disposal of such cases by revealing the presence or absence of serious disproportion on former occasions, further help may be derived from examination under anaesthesia and from radiological examination.

\section{History of Toxaemia.}

Toxaemia most commonly arises during a first pregnancy, although it may appear for the first time in a second or later cycle. Once the condition has appeared, it is prone to recur, and recurrence is the more likely if the initial attack has been severe or prolonged. When such a history is obtained the details of the initial attack should be ascertained and special care taken to detect the appearance of albuminuria, hyperpiesia, and other prodromata in order that steps may be taken to deal with the situation. The methods of treatment are simple-rest, diet, aperients, but they must include a daily record of the amount of urine, degree of albuminuria, blood pressure, and laboratory investigations such as blood urea estimations and renal efficiency tests may also be required. These observations and investigations cannot be conveniently carried out in domiciliary work, but they are necessary to accurate diagnosis and treatment, and without their aid it is impossible to determine the progress of the patient, the response to treatment, and the need for termination of the pregnancy. All multiparae with prodromal signs of toxaemia demand the help of a consultative clinic, and the resources of a fully equipped Maternity hospital-there is no place for them in domiciliary work.

\section{History of Organic Disease.}

Most organic diseases form a harmful liaison with the changes set up during pregnancy and the strain imposed by labour but two main distinctions may be drawn between the primigravida and the multipara in regard to the quality of the vicious circle thus brought into action. First, since many organic lesions are chronic, their influence is felt throughout each of a series of pregnancies and labours, and as many diseases are also progressive, their effects will tend to increase with parity. An organic disease is therefore generally less advanced when the patient is a primigravida than when she becomes a multiparous woman. This general principle is however modified by the nature of the disease and its mode of action upon the pregnant and parturient state.

Chronic nephritis, for example, is a progressive disease which leads eventually to renal failure, and therefore it may be expected to advance with age and parity, and indeed to be enhanced by these factors unless steps are taken to avoid conception or nature intervenes to 
produce sterility or abortion. The primigravida, however, is specially prone to toxic albuminuria, a condition which forms a symbiosis of peculiar gravity with an organic lesion of the kidney, and therefore a woman with kidney disease may suffer more from this condition in her first pregnancy than in a subsequent one.

Heart disease is also a chronic and often progressive condition, and the patient will have a better cardiac reserve as a primigravida than as a multipara, but the quality of the vicious circle is due to factors which are greatly affected by the parity of the patient. These factors, which are progressive in their action during pregnancy and reach their climax at and immediately after labour, are far more potent in a primigravida than in a multiparous woman, so that here again the effects of age are anticipated as it were by the peculiar stigmata of the primigravida. She is most vulnerable to the pressure effects of pregnancy, because the firm tone of her parietal and pelvic muscles resisis the growing uterus, and in so doing deflects its pressure to the diaphragm and the organs in contact with that structure, the heart and lungs; and for similar reasons the risk to the damaged heart during delivery is far greater in the primigravida. In the multipara, muscle tone being much less, the abdominal wall and the pelvic floor yield to the increasing pressure and thus relieve the diaphragm of the strain: as a result, the multipara, if her cardiac reserve is not seriously depleted beforehand, often shows surprisingly little distress during pregnancy, and also during labour, for it has been noted that such patients have easy deliveries. For these reasons heart disease must be regarded as a less formidable complication to a multipara than to a primigravida in whom the first reproductive cycle may lead to complete decompensation and render the patient unfit for further reproduction. These principles must be borne in mind whenever the clinician is confronted with the general problem of organic disease and pregnancy. The vicious circle must be analysed into its component parts and their mode of action upon each other duly considered in regard to the age and parity of the patient. This will enable the practitioner to evaluate the situation and to take the necessary steps for the treatment and disposal of the case.

\section{GROUP 4.-THE OLD MULTIPARA.}

Repeated childbearing and advancing age produce changes which increase the risks of childbearing, and which serve to distinguish the old multipara not only from the primigravida, but also from the young parous woman. These changes may be seen in comparative youth after but two or three labours, or they may not be found in a patient of forty who has had ten deliveries, and there can be no doubt that many factors influence their time of onset and rate of development. The effect's or stigmata thus produced may be summarised under two headings-(I) loss of muscle tone, and (2) excessive physiological wear and tear.

\section{Loss of Tone.}

Anaemia, calcium depletion, faulty involution and other changes of an obscure nature which follow or may follow repeated childbearing lead to loss of tone in the uterine, parietal, cardiac and skeletal muscles, and cause malpresentations of the foetus, uterine inertia, and postpartum haemorrhage.

\section{Physiological wear and tear.}

Advancing age is associated with the appearance of changes that reduce the functional capacity of the bodily organs and render the individual progressively less fit for great or sustained effort. The influence of age upon the parous woman is to make her less.fit to meet the exceptional demands imposed by pregnancy upon the excretory organs and by labour upon the musculature and heart; the general effect upon the old multipara, who is otherwise healthy, is to lower her general resistance, to reduce her capacity for work, and to render her more susceptible to fatigue and shock. If these effects appear sooner or later in healthy women, it is only to be expected that they will appear earlier in women suffering from diseases which make a progressive encroachment upon the reserve power of the affected organs. As we have seen, the process of reproduction has a potent influence upon many diseases by enhancing their effects and by stimulating their progress, and hence the stigmata of excessive parity must form with organic disease a dangerous combination that becomes more formidable with advancing age. There are therefore many reasons for regarding the old multipara as a 
special problem and giving to her that special attention which does not at first sight appear to be necessary. This aim is most easily achieved by sending all old multiparae to hospital where as an out-patient during pregnancy, and as an in-patient during labour they may be assured of the special nursing, medical and laboratory resources which their condition demands, and which only an institution can provide. Many old multiparae will not take this advice, and it is therefore necessary to refer briefly to the domiciliary treatment of some of the more common complications which may arise in such cases.

\section{Concealed Ante-Partum Haemorrhage.}

For some as yet unknown reason this toxic condition has a peculiar affinity for multiparae - of fifty cases of A.P.H. recorded by the late Gordon Ley, only eight occurred in primigravidae and no less than twenty-one in women with five or more pregnancies: age also is certainly a contributory factor, as ten of these women were forty years of age or more. The treatment of such women is quite clear, and so long as the gravity of the shock is realised most of them will get better if they are treated at home: indeed, removal to hospital before the shock has been combated is a dangerous procedure calculated to increase the mortality of the condition. Moreover, removal is unnecessary because radical interference is no longer practised (Caesarean section for a patient in profound shock being an extremely hazardous and indeed unjustifiable operation), and the proper conservative measures can be applied just as easily at home-these include:

I. Morphia, warmth, salines, posture, fluids, perhaps blood.

2. Obstetric interference forbidden until the uterus resumes activity: it may well be disastrous if carried out prematurely.

3. When the shock has passed off, the patient may be moved to hospital, or help may be obtained from the "Flying Squad"-but special obstetric assistance is rarely necessary as these cases generally deliver themselves without difficulty.

4. Throughout the case the greatest attention must be paid to the general condition of the patient and obstetric interference-even such a simple procedure as rupturing the membranes-should be avoided until there is clear evidence of resumption of uterine activity.

\section{Secondary Uterine Inertia.}

Of 200 cases of craniotomy taken from the reports of six Maternity hospitals, 24 of the patients were between 40 and 47 years of age, and 44 of them had had 6 to 9 children, including Io women with II to I6 children each. Few of the patients showed any gross mechanical defect, and most of them gave a deceptively innocent past history-all of them coming into the class of the old multipara. The mechanical difficulty demanding such radical treatment as craniotomy was due to a combination of those factors which have already been described as the stigmata of age and parity-a large or relatively large baby (hypermaturity appears to be associated with high degrees of parity), a malpresentation such as R.O.P.- an old rigid, perhaps partially decalcified pelvis (implying functional if not anatomical contraction) in combination with a defective musculature incapable of producing power sufficient to correct the malpresentation and to overcome the obstacle. In such cases the myocardium, too, is affected and incapable of sustained effort, and unless steps are taken to overcome the obstetric difficulty, cardiac as well as uterine exhaustion sets in and the patient may die of cardiac failure. The treatment must be adapted to the circumstances of the case and the time and place at which the patient first comes under observation, but the following methods may be employed:

I. Recognition of the type during pregnancy-investigation of blood picture, renal efficiency, etc., with appropriate counter measures . . . diet, iron, blood transfusion . . . designed to improve the general and muscular tone.

2. Admission to hospital before labour for assessment of mechanical situation, and with a view to preventive measures such as correction of malpresentation, prevention of hyper-maturity by induction of premature labour, and even Caesarean section for chosen cases.

3. If the patient is in labour when she first comes under skilled observation, it is important to recognise that the patient conforms to the type under discussion, and it may be 
wise to move her to hospital if her condition permits and the facilities are easily available. When for any reason this is impracticable, measures must be taken to conserve the cardiac reserve, and arrangements made to deliver the patient by the quickest and least shocking method. Sometimes sedatives may be sufficient to tide the patient over the fatigue of the first stage, and forceps applied as soon as the necessary indications are fulfilled, whilst version to breech may sometimes be the method of choice. There should be no hesitation about performing craniotomy when the child is dead, or when in the presence of an insuperable obstacle Caesarean section is contra-indicated or impracticable.

\title{
Third Stage Bleeding.
}

Bleeding during the third stage is more common and more dangerous among old multiparae than any other type of case-whereas traumatic haemorrhage is restricted almost entirely to primigravidae and requires no comment here. The common atonic type is due to two factors -an irregular attachment of the placenta to the uterus whereby one part of the placenta is too lightly attached and the remainder too firmly adherent to the placental site, and to defective tone of the uterine muscle which renders it incapable of producing and sustaining the strong contraction necessary to complete the detachment of the placenta. Treatment may again be preventive, iron, calcium hormones, and other measures may be given during pregnancy to improve the tone of the uterus, and if the old multipara type is recognised, arrangements made for admission before labour. During the third stage of labour the uterus should not be "hustled" during the.period of temporary relaxation which follows delivery. Massage at this resting stage is unnecessary because the placenta has not yet separated from any part of the uterine wall, and it is positively harmful because it may lead to separation at areas of light attachment and thus actually encourage bleeding. When bleeding occurs the following procedures must be carried out forthwith:

I. The patient must not be moved until the bleeding has been controlled and the placenta has come away or been removed manually. The temptation to send the patient to hospital is great, but it must be resisted.

2. The fundus must be massaged gently in order to stimulate general contraction of the uterus.

3. If bleeding persists, Crede's method of expression must be tried-i.e. squeezing the fundus without depressing the organ, care being taken to avoid injury to the sensitive ovaries.

4. Crede's method must not be employed more than twice because it causes shock, and if the attempt is unsuccessful the patient is left worse off than she was beforehand: repeated attempts to express the placenta may render the patient unfit to stand any further active treatment.

5. If Crede's method fails-then manual removal must be carried out, and so long as rigid antisepsis is maintained, and the patient is not deeply shocked, this method is less dangerous than repeated and futile attempts at expression.

6. Antishock measures are essential during and after the control of the bleeding-warmth, posture, fluids, etc.

\section{ANTE-PARTUM HAEMORRHAGE}

\author{
By GLADYS H. DODDS, M.D., F.R.C.S., F.R.C.O.G. \\ (Assistant in the Obstetric Unit, University College Hospital)
}

\section{THE CLINICAL DIAGNOSIS}

Haemorrhages in the later weeks of pregnancy, no matter how slight, are a source of anxiety. The first haemorrhage is often slight, there may be no further bleeding, or several days or weeks may elapse before the bleeding recurs. This recurrent haemorrhage may again be slight, but it 